\title{
Left ventricular function plays a role in cognitive ageing
}

\author{
E. E. van der Wall
}

Published online: 12 October 2011

(C) The Author(s) 2011. This article is published with open access at Springerlink.com

Ageing affects and is also being affected by various physiological processes in the human system, in particular the brain $[1,2]$. In patients with severe cardiomyopathies, left ventricular ejection fraction (LVEF) is related to abnormal brain ageing, including cognitive impairment, structural neuroanatomical abnormalities, and increased risk for Alzheimer's disease. Cognitive impairment diminishes and cerebral blood flow increases by more than $50 \%$ after heart transplantation because of improvement in cardiac function. Therefore, a reduced LVEF may influence cerebral perfusion homeostasis and contribute to clinical brain injury.

To further investigate this issue, Angela Jefferson and coworkers (Boston University School of Medicine, Massachusetts, USA) analysed brain magnetic resonance imaging (MRI) and cardiac MRI studies as well as the results of neuropsychological examinations in 1114 Framingham Heart Study Offspring patients, aged 40 to 89 years, mean age $67 \pm 9$, mean LVEF $67 \%$, 54\% women (published online in the American Journal of Cardiology, September 1, 2011). In the absence of end-stage heart disease, it was hypothesised that LVEF would be associated with preclinical brain MRI and neuropsychological markers of ischaemia and Alzheimer's disease in the community. Neuropsychological and neuroimaging markers of brain ageing were related to cardiac MRIassessed LVEF. Cardiac MRI was used for LVEF assessment, being the most accurate approach to determine left ventricular function in a variety of cardiac diseases [3-6].

E. E. van der Wall $(\bowtie)$

Department of Cardiology, Leiden University Medical Center, PO Box 9600, Leiden, the Netherlands

e-mail: e.e.van_der_wall@lumc.nl
It was shown that a relatively low LVEF (less than 62\%) was associated with lower mean cognitive performance including verbal and visuospatial memory, executive functioning, and visuoperceptual abilities.

The mechanisms underlying associations between a lower LVEF at rest and abnormal brain ageing are unknown. Despite autoregulatory mechanisms, cerebral blood flow values are low in heart transplantation candidates but return to normal after heart transplantation. Disruption of cerebral perfusion may contribute to clinical or subclinical brain injury by propagating or exacerbating cerebrovascular disease, including changes in microvessel structure, expression of vascular cell receptors, microvessel permeability changes, and vascular remodelling. Chronic cerebral hypoperfusion puts the brain at risk for amyloid deposition resulting in neuronal death in transgenic amyloid mice.

An unexpected observation was that also participants in the highest LVEF ranges (more than 73\%) had poorer cognitive performance. Whereas a normal left ventricular function may be good for brain health, very high LVEFs may also correspond to subtle cognitive impairment. The authors concluded that patients with a low LVEF performed significantly worse on several cognitive tests than patients with a moderate LVEF. However, there was no linear association since U-shaped curves also showed an association between the highest LVEF levels and cognitive ageing.

In general, the observation that a lower LVEF is associated with abnormal brain changes extends previous research examining patients with severe cardiomyopathies [7], showing that a reduced LVEF was associated with impaired memory and reasoning. More research is needed to understand the mechanisms accounting for the heartbrain associations. The present study emphasises the need for accurate measurement of LVEF to be aware of cerebral 
(mal)function in cardiac patients, in particular those with reduced left ventricular function.

Open Access This article is distributed under the terms of the Creative Commons Attribution Noncommercial License which permits any noncommercial use, distribution, and reproduction in any medium, provided the original author(s) and source are credited.

\section{References}

1. Van der Wall EE. The brain-heart connection: a round trip. Neth Heart J. 2011;19:269-70.

2. Torn M, Bollen WL, van der Meer FJ, et al. Risks of oral anticoagulant therapy with increasing age. Arch Intern Med. 2005; 165:1527-32.
3. Matheijssen NA, Louwerenburg HW, van Rugge FP, et al. Comparison of ultrafast dipyridamole magnetic resonance imaging with dipyridamole SestaMIBI SPECT for detection of perfusion abnormalities in patients with one-vessel coronary artery disease: assessment by quantitative model fitting. Magn Reson Med. 1996;35:221-8.

4. de Roos A, Doornbos J, Luyten PR, et al. Cardiac metabolism in patients with dilated and hypertrophic cardiomyopathy: assessment with proton-decoupled P-31 MR spectroscopy. J Magn Reson Imaging. 1992;2:711-9.

5. Nollen GJ, Groenink M, Tijssen JG, et al. Aortic stiffness and diameter predict progressive aortic dilatation in patients with Marfan syndrome. Eur Heart J. 2004;25:1146-52.

6. van der Wall EE, Reiber JH. Assessment of left ventricular function: visual or quantitative? Int J Cardiovasc Imag. 2011;27:573-7.

7. Jefferson AL, Himali JJ, Beiser AS, et al. Cardiac index is associated with brain aging: the Framingham Heart Study. Circulation. 2010;122:690-7. 